\title{
Partial analysis of high-way factorial designs ${ }^{\prime}$
}

\section{NORMAN H. ANDERSON, DEPARTMENT OF PSYCHOLOGY, UNIVERSITY OF CALIFORNIA, SAN DIEGO, Calif. 92037}

Inclusion of several variables in a design is often desirable, but each added variable doubles the number of significance tests in the routine analysis of variance. This note discusses a procedure of partial analysis in which interactions, primarily those involving minor variables, are omitted from the analysis when prior evidence indicates that they are probably negligible. The rationale of this procedure is given, and its use illustrated in a model experiment. Specific recommendations for handling systematic sources and error terms are given.

Careful experimenters often face problems that their more casual colleagues avoid. One of these arises when several variables are included in the experimental design. Routine analysis of a 5-way design. for instance, yields 30-odd F-tests. Since one or two of these could be expected to be significant by chance alone, any one significant result is often unconvincing. This is especially true when prevailing knowledge suggests that the true effect is negligible.

Under these circumstances, the person who finds a significant statistical interaction may have problems in reporting his , esults. He naturally feels under some obligation to describe the interaction in terms of the data means and to attempt a substantive interpretation. Just describing an interaction can be complicated, however, and it is doubly awkward when it is suspected of being merely chance fluctuation. Sometimes it is pussible to dismiss the matter briefly, but dismissing significant results is not done easily, even when one is moderately sure they are not real.

The purpose of this paper is to discuss a procedure of partial analysis of multifactor designs. Partial analysis avoids the above problems with interactions by simply not testing them when prevailing knowledge indicates that they are probably negligible. Though it may seem odd to argue for neglecting certain statistical. tests, the present proposals are little more than common experimental sense and occasional applications of partial analysis can be found in the literature. Accordingly, this article may be considered primarily expository, aimed at unifying and systematizing the relevant considerations.

\section{ILLUSTRATIVE EXPERIMENT}

Application of partial analysis depends heavily on empirical aspects of the proposed experimentation, and it is instructive to consider its use in an actual case. In this section, therefore, a carefully designed experiment by R. L. Erickson (1965) in which this procedure was employed will be used to illustrate the main considerations.

Erickson's experiment had an extremely simple purpose: to test whether isolation of stimulus or of response was more effective in paired-associate learning. This isolation, or von
Restorff effect, bears on the theoretical analysis of verbal learning, particularly on interference theory (Erickson, 1963, 1965). Here, of course, the main interest is on method.

Erickson's Ss learned eight pairs in which stimulus and response were all two-consonant syllables. All items were printed in black except the one isolated syllable which was red. Training was given on a memory drum at a 2:2:1 sec time sequence for 24 presentations of the list. The main dependent variable was the difference in ease of learning isolated and nonisolated pairs.

At an abstract level, this experiment is straightforward. Indeed, only two groups are required, one with the stimulus isolated, the other with the response isolated. At the practical level, Erickson ended with six factors in his design, none of which could have been omitted without diminishing the quality of the work. Only one of these was of major interest, and three were minor variables with little substantive importance. The choice of statistical analysis, however, depends on the nature of these minor variables.

\section{Experimental design}

The main variable was stimulus-vs-response isolation. For half the Ss, the stimulus syllable of one of the pairs was in red; for the other half, the response syllable of the pair was in red. The primary experimental question was whether these two isolation conditions would produce different learning rates.

There were also two subsidiary variables with direct psychological interest. The first of these was amount of isolation, a possible determinant of the size of the von Restorff effect. At one level, the isolated item was in red but in the same type as the black items: at the other level, the isolated item was not only red, but also appeared in a larger and different style of type.

The second subsidiary variable arose from a methodological concern. In the usual paired-associates procedure, the stimulus item appears before but terminates at the same time as the response item. This greater stimulus duration could produce stimulus-vs-response effects confounded with the main variable. Although there was some reason to believe that this was not a serious problem, Erickson felt that the design should not ignore it. Accordingly, two stimulus exposure conditions were used. One was based on the standard presentation procedure in which the stimulus alone was visible for $2 \mathrm{sec}$, and both stimulus and response for an additional $2 \mathrm{sec}$. In the other exposure condition, the stimulus was visible for $2 \mathrm{sec}$ only, followed by the response alone for the next $2 \mathrm{sec}$. This procedure would not necessarily solve the problem, if one existed, but it did set out a test signal for possible danger.

Two of the minor variables had to do with item replication and balancing. All lists were constructed from the same eight pairs, and of these, four were chosen as critical pairs of comparable difficulty on the basis of pilot data. Thus, the eight pairs yielded four lists differing in which pair was isolated. This item replication extends the generality of the results and, more 
importantly, it allows the use of a within-S dependent variable as noted below.

The second minor variable balanced items across stimulus and response positions. From the above four lists another four were constructed by interchanging the stimulus and response items within every pair. This balancing across Position is needed to factor item differences out of the main comparison.

The final variable was Experimenters. The two undergraduate assistants who ran the $\mathrm{Ss}$ were included as a factor in the overall design.

The complete design was thus a $2^{5}$ by 4 factorial. There were $128 \mathrm{Ss}$ in the experiment, one $\mathrm{S}$ in each cell of the design. Random assignment of Ss was used except for the E-variable. Each $\mathrm{E}$, however, $\operatorname{ran}$ his Ss as they arrived in a prerandomized order of the 64 conditions.

\section{Dependent variable}

The main dependent variable was the isolation score. This was computed for each $\mathrm{S}$ by multiplying by three the total number of correct responses to the isolated pair, and subtracting from this the total number of correct responses to the three comparable nonisolated pairs. This score is a direct and integral measure of the isolation effect. A score of zero would indicate no benefit from the isolation.

The use of this difference score allows each $\mathrm{S}$ to serve as his own control. This will decrease the variability under certain conditions, noted below, that could reasonably be expected in this experiment. The use of this difference score makes necessary the use of the Item variable as already stated.

\section{POSSIBLE STATISTICAL ANALYSES}

The main purpose of this paper is to discuss some of the principles that underlie the choice of statistical analysis for designs like the above. The relevant considerations will first be illustrated by reference to this experiment, and a more general set of recommendations will be given in the next section.

The variable of Experimenter will be ignored except where explicitly considered. This simplifies the discussion and does no violence to the data since $E$ had no apparent effect. On this basis, the design becomes a $2^{4}$ by 4 factorial with two $S$ in each of the 64 cells.

One technical feature of these analyses has some incidental interest. Because of the definition of the dependent variable, it is of interest to test whether its overall mean is different from zero. This can be done within the regular analysis (e.g., Anderson, 1960). The source, "Mean," is added to the table and tested against the error term in the usual way. This source has $1 \mathrm{df}$ and its $S S$ is just $(\Sigma X)^{2} / N$, the ordinary correction term. This $F$ tests the null hypothesis that the overall true mean is zero. (Ordinarily this question has no interest and is not tested which is why ANOVA tables usually have only $\mathrm{N}-1 \mathrm{df}$.)

\section{Complete analysis}

Routine application of analysis of variance to this 5-way design would produce the summary table given at the left side of Table 1. The $F$ of 131.19 for Mean shows that the isolation effect. averaged over all conditions, was reliable. This effect was greater with stimulus than with response isolation, and the $F$ of 12.34 shows that this difference was reliable. The main purpose of the experiment was thus fulfilled, and the nonsignificance of the other main effects helps solidify the main results. There are in addition two significant interactions whose present relevance is mainly to illustrate the objections to the complete analysis.

The main objection to this routine analysis is that it potentiates unduly many Type $\mathbf{I}$ errors. If all 26 interactions were zero in truth, testing at the customary .05 level would necessitate an expectation of one-plus falsely significant results per experiment. Against that expectation, the reliability of the two significant interactions in Table 1 becomes uncertain. This uncertainty is increased when prior evidence gives grounds for believing that many of these interactions will be negligible. For instance, consider the Item by Size interaction. The pilot data, together with the general laboratory experience available when the experiment was planned, indicated that Item differences would be relatively small. Existing knowlege would also suggest no marked effect of Size. Under these circumstances, a significant Item by Size interaction would have been difficult to credit and would reasonably be considered a likely Type I error. But if this argument is correct, there is not much point to making the test in the first place

Similar reasoning holds for the higher-order interactions involving Item and Size, and for various other interactions including, perhaps, the two that were significant in the present analysis.

This outlook is reinforced by a more practical consideration. A person who did the complete analysis as given would feel under some obligation to describe the significant ltem by S-R by Time interaction in terms of the data means. Frequently, however, it is difficult just to describe a three-way interaction, especially when it makes no particular sense. This difficulty is felt all the more when prevailing knowledge suggests that the result is merely chance anyway.

\section{Three-way analysis}

Faced with the above problems, one might simply omit the minor variables in the statistical analysis since they are the main source of the problems. This leads to the 3-way analysis in the center of Table 1 to which the above objections hardly apply. The number of tests is reduced from 32 to 8 , and all involve variables with substantive interest. The two significant interactions in the complete analysis have vanished. This approach is not infrequent; it is adopted every time a variable is counterbalanced in the design but ignored in the data analysis.

The main objection to this 3-way analysis is that it goes too far. Applied to the complete experiment, it would provide no test of the equivalence of the two Es. Such a test would be desired by Erickson as a check on his two assistants, and by the reader as a check on Erickson's technique. Sinilarly, an evaluation of Item differences could be useful in planning further work even though it has little substantive value.

In addition, the 3-way analysis tends to inflate the error term and decrease the power of the test. Any effects of ltem, for instance, are in the data regardless of the analysis employed. If they are not extracted, they necessarily end up in the error term whose value is thereby increased on the average, though not necessarily in every set of data.

\section{Suggested partial analysis}

The right side of Table 1 presents a partial 5-way analysis that strikes a balance between the two above. The sources selected for testing include all the sources from the 3-way analysis, the main effects of the three minor variables, and all 2-way interactions 
involving stimulus-vs-response isolation. This analysis is quite similar to that adopted by Erickson.

This is. of course. a nearly minimal analysis since only a couple of the listed sources could reasonably be omitted. On the other hand, other investigators might include a few additional sources. Some of these decisions might be close, but none would seem of great importance.

\section{PARTIAL ANALYSIS}

\section{Rationale}

The problems that have been illustrated in the Erickson experiment now need to be treated more generally. Accordingly, the basic rationale and the safety features of partial analysis will be discussed, and these will then provide the basis for a set of practical recommendations.

The rationale of partial analysis is simple and straightforward. In certain experiments. existing knowledge will provide a reasonable basis to expect thilt certain interactions will be negligible. If this expectation is correct. testing such interactions is undesirable since it merely potentiates Type I errors. In the partial analysis procedure, such interactions are not tested. Since it makes little practical difference whether the null hypothesis is exactly or nearly true, the term Type I error will here include both cases.

Whether partial analysis is applicable in any experiment depends on the body of knowledge prevailing when the experiment is designed. Although prevailing knowledge is often vague, ill-defined, and uncertain, it still provides a basis for action whose validity is a matter of general experience. In the above experiment, it would be impossible to find a firm and explicit basis for concluding that the Experimenter by ltem interaction was negligible. Presumably, however, few workers in verbal learning would disagree with this assessment and, presumably, it is correct. In general, clear and solid evidence will seldom be available, and the decisions will need to be made on the basis of accumulated knowledge of varied relevance. Of course, some degree of presumptive agreement is needed before an interaction may be neglected in a public report. Theoretical prediction and personal hope do not themselves form an adequate basis for partial analysis.

Existing knowledge, or strictly, one's own share of it, may be

Table 1

Comparison of Three Possible Analyses of Erickson Experiment

Complete Analysis Three-way Analysis

Partial Analysis

\begin{tabular}{|c|c|c|c|c|c|c|c|c|}
\hline Source & $\mathrm{df}$ & $\mathbf{F}$ & Source & $\mathrm{df}$ & $\mathrm{F}$ & Source & $\mathrm{df}$ & $\mathbf{F}$ \\
\hline Mean & 1 & $131.19^{*}$ & Mean & 1 & $131.86^{*}$ & Mean & 1 & $131.19^{*}$ \\
\hline Stimulus-Response & 1 & $12.34 *$ & Stimulus-Response & 1 & $12.41^{*}$ & Stimulus-Response & 1 & $12.34 *$ \\
\hline Size & 1 & .86 & Size & 1 & .86 & Size & 1 & .86 \\
\hline Time & 1 & 3.35 & Time & 1 & 3.37 & Time & 1 & 3.35 \\
\hline Item & 3 & 1.58 & $S R \times S$ & 1 & .74 & Item & 3 & 1.58 \\
\hline Position & 1 & 2.05 & $S R \times T$ & 1 & .67 & Position & 1 & 2.05 \\
\hline$S R \times S$ & 1 & .74 & $\mathrm{~S} \times \mathrm{T}$ & 1 & .78 & $S R \times S$ & 1 & .74 \\
\hline SR $\times T$ & 1 & .66 & $S R \times S \times T$ & 1 & .43 & $S R \times T$ & 1 & .66 \\
\hline SR $\times I$ & 3 & .92 & Error MS & 120 & $(426.49)$ & SR $\times I$ & 3 & .92 \\
\hline$S R \times P$ & 1 & $4.36^{*}$ & & & & $S R \times P$ & 1 & $4.36^{*}$ \\
\hline$S \times T$ & 1 & .78 & & & & $S \times T$ & 1 & .78 \\
\hline$S \times I$ & 3 & .87 & & & & $S R \times S \times T$ & 1 & .43 \\
\hline$S \times P$ & 1 & .04 & & & & Pooled Residual MS & 48 & $(370.63)$ \\
\hline$T \times I$ & 3 & .19 & & & & Error MS & 64 & $(428.68)$ \\
\hline$T \times P$ & 1 & .06 & & & & & & \\
\hline$I \times P$ & 3 & .42 & & & & & & \\
\hline$S R \times S \times T$ & 1 & .43 & & & & & & \\
\hline SR $\times S \times I$ & 3 & .86 & & & & & & \\
\hline$S R \times S \times P$ & 1 & .05 & & & & & & \\
\hline SR $\times T \times I$ & 3 & $3.71 *$ & & & & & & \\
\hline$S R \times T \times P$ & 1 & .19 & & & & & & \\
\hline$S R \times I \times P$ & 3 & .60 & & & & & & \\
\hline$S \times T \times I$ & 3 & .78 & & & & & & \\
\hline$S \times T \times P$ & 1 & .56 & & & & & & \\
\hline$S \times I \times P$ & 3 & .80 & & & & & & \\
\hline$T \times I \times P$ & 3 & 1.58 & & & & & & \\
\hline$S R \times S \times T \times I$ & 3 & 1.36 & & & & & & \\
\hline$S R \times S \times T \times P$ & 1 & .00 & & & & & & \\
\hline$S R \times S \times I \times P$ & 3 & 1.10 & & & & & & \\
\hline$S R \times T \times 1 \times P$ & 3 & .26 & & & & & & \\
\hline$S \times T \times I \times P$ & 3 & .04 & & & & & & \\
\hline$S R \times S \times T \times I \times P$ & 3 & .98 & & & & & & \\
\hline Error MS & 64 & $(428.68)$ & & & & & & \\
\hline
\end{tabular}

${ }^{*} p<.05$ 
In coror in ang given instance. The decision not to tast might then suppress an inportant finding. Fortunately, partial antaly sis has a safety feature because serious interactions seldon occur in practice unless the variables have substantial main effects. In Erickson's experiment, large Item differences would have caused concern and led to a more detailed scrutiny of the data. regardless of whether the Item by S-R interaction was significant. Again, a large difference between Experimenters would itself have raised serious questions about the validity of the data. On the other hand, if the observed effects of the minor variables are small or moderate, this constitutes some practical justification for having ignored their interactions. In a similar manner, 2-way interactions serve as warning signals for higher-order interactions.

Two-way interactions can occur without main effects of either variable. This does not happen very often, however, especially with minor variables. Although the safety feature is not guaranteed, therefore, it does offer substantial protection.

To this, it should be added that an interaction may be real, yet devoid of interest. As is well known, interactions may reflect nothing more than the choice of dependent variable or scale of measurement. Many of the interactions reported in the literature seem to be nothing more than floor or ceiling eftects of no particular value. Again, interactions obtained with a time score may disappear when time is transformed to speed, or vice versi. Some further discussion of this matter in various connections can be found elsewhere (e.g., Anderson, 1961, 1962, 1963; Anderson \& Jacobson, 1968: Green \& Tukey, 1960; Prokasy, 1962; Sidowski \& Anderson, 1967; Winer, 1962). The possibility that partial analysis may cause some real interactions to be overlooked should be viewed in this perspective.

The next two subsections give practical recommendations for applying partial analysis. Error sources involve different considerations than systematic sources and are treated separately. These recommendations should be understood relative to the conditions under which partial analysis is most appropriate. The main condition, of course, is that certain factors should represent minor or subsidiary variables. Also, when there are only two or three factors in the design, there is little need or opportunity to apply partial analysis. Finally, it is assumed here that none of the factors, except perhaps Ss in repeated measurements designs, are "random" factors, in which the levels of the factor are selected at random.

Systematic sources

Formalization of the above considerations leads to the following practical recommendations about testing systematic sources. These are meant only as general guidelines; each experimental design needs to be evaluated in its own right relative to the background information.

(1) Test all main effects.

(2) Test all interactions involving only major variables.

(3) Test 2-way interactions involving a major and a minor variable.

(4) Test any remaining interaction that seems warranted from considerations of potential importance and probable magnitude.

(5) Decide what sources will be tested before looking at the data.

These recommendations lead to essentially the same partial analysis of the Erickson experiment as given in Table 1. The first four points are in line with the previous discussion and need no funther remark. The lifth point has its usual double purpose of avoiding temptation to suppress an unpleasant feature of the data, and of avoiding ensnarement in chance results.

To the above should be added a criterion of simplicity of exposition. The reader of an article needs to evaluate the soundness of the design and analysis, and his job should be eased as much as possible. If testing a few additional interactions will simplify the description of the procedure, or the interpretation of the results, this should be done.

\section{Error sources}

There are two main cases that arise in the treatment of the error suurces. These depend on whether or not it is necessary to pool interactions to obtain an crror term.

The first case is illustrated in the recommended analysis of the Erickson experiment in Table 1 . The SSs for the listed systematic sources are computed in the usual way. Since the E-variable is being ignored, there are two scores in each of the 64 cells of the design, resulting in $64 \mathrm{df}$ for error. The SS for error is computed from the usual rule: $\Sigma X^{2}-\Sigma T_{j}^{2} / 2$, where $X$ is a raw score, and $T_{j}$ a cell total (here based on two scores)

This leaves open the disposition of the systematic sources that are not being tested. One possibility is to pool these interactions with error, which would be done simply by adding the SSs and the $\mathrm{dfs}$ for the sources being pooled. Any interaction whose population value is zero provides an unbiased estimate of variability; pooling it would increase the reliability of the error term and so provide a more powerful test. However, if the interaction is not zero, then the pooling would tend to increase the error mean square which would decrease the power of the test.

In the present illustration, the regular error term has a substantial number of $\mathrm{df}$, and the possible increase in power from pooling is very small. Accordingly, the untested interactions have been pooled to form a Pooled Residual whose SS is simply $\Sigma \mathrm{T}_{\mathrm{j}}^{2} / 2$ minus all systematic SSs, including Mean. This procedure is probably preferable as long as the regular error term has more than, say, $30 \mathrm{df}$. The potential gain from pooling interactions with error rises as error df decline, and such pooling would become very important if error $\mathrm{df}$ dropped below 10 .

The second case that arises in treating the untested interactions is one that actually obtained in the Erickson experiment. So far in this discussion, the factor of Experimenter has been ignored in the analysis. When this factor is included, the design is a 6-way factorial with one score per cell. As a consequence, no estimate of within-cell variability is available, and an error term can be obtained only be pooling various systematic sources.

Pooling interactions for error raises further problems than not testing them since the error term affects all the tests that are made. Expanding the design so that the cell size is reduced to one thus needs careful consideration. In Erickson's experiment however, there would seem to be no cause for concern.

In this connection. it may be remarked that variables that are inherent in the experiment do not create the same degree of difficulty. The effects of any such variable are necessarily in the data; the only question is whether they end up in the error term or in the systematic sources. The investigator who includes such a variable in lis design and evaluates its main effect is thereby extracting one component of variability out of the error term. If the variable has a direct effect, this procedure will decrease the 
error mean square even if it is constituted of the interactions of that variable, as with a cell size of one. Individual differences are the prime source of inherent variables, and incorporating them in the design and analysis deserves more consideration than has been given (e.g., Anderson, 1959).

In the present experiment, perhaps the most efficient and conceptually simplest procedure would be to pool the 4, 5-, and 6-way interactions for error. This would yield $54 \mathrm{df}$ for error which is ample; inclusion of the lower-order interactions would probably tend to increase the error mean square slightly without adequate compensation from the increase in $\mathrm{df}$.

This last procedure is quite demanding if the calculations are done with a desk machine. Erickson actually pooled all the untested interactions to obtain an error mean square on $104 \mathrm{df}$. With hand computation, this complete pooling procedure is especially simple. The SS for error is simply $\Sigma \mathrm{X}^{2}$ minus the SS for all tested sources. (The SS for Mean should be subtracted whether or not it is tested.)

One incidental aspect of the 6-way analysis has some methodological interest. When Experimenter is included in the analysis, its mean square turns out to be 7.51 , very much smaller than the error term. This suggests that perhaps the two Es were closer than likely by chance. Though this was hardly possible in Erickson's experiment, Es in other situations might influence one another's results in various ways. The hypothesis that the difference between E-means is unduly small can be tested simply by inverting the usual F-ratio, inverting the $\mathrm{df}$, and proceeding as usual (Hoel, 1962). Here this would yield an F of 52, not even close to the .05 critical value of 252 on $54 / 1 \mathrm{df}$. Accordingly, there is no reason to think that the Es were more alike in their output than chance would allow.

\section{Alternative designs}

In experimental design, directness and simplicity are fundamental virtues. The present concern with statistical analysis of high-way designs should not obscure the desirability of avoiding the problem at the basic design level. Accordingly, it is instructive to ask whether the experiment might not have been usefully simplified.

The most obvious way to simplify would be to omit one of the factors in the design. The use of two degrees of isolation, for instance, was not essential and this variable could have been omitted without harm. However, including it entails relatively little experimental cost, and need not entail much statistical effort. This variable is not without psychological interest, and its inclusion added to the value and solidity of the results.

The variable of Experimenter presents a somewhat different problem since it was necessary to use two Es. If two Es are used, then they are de facto a variable in the experiment, and any Eeffect will be in the observed data. The only question is whether or not Experimenter is included as a formal factor in the design so that a statistical test is possible. It is certainly preferable to include it, though failure to do so would probably not be serious in most verbal learning experiments. In other areas, such as animal learring (e.g., Brogden, 1962; Logan, 1960), or social psychology (e.g., Rosenthal, 1966), experimenter effects would need to be considered more seriously.

A third variable that might be omitted is Item. Only one critical pair is strictly necessary, with the stimulus syllable or the response syllable being isolated for half the Ss. This would, however, disallow the above difference score as the dependent variable since its use depends on the balancing provided by the Item factor in the design. Number correct on the isolated pair only would still be a valid dependent variable, but would waste the information contained in the performance on the nonisolated pairs. Indeed, it is straightforward to show in the present case that the difference score has less variability and hence gives greater power as soon as the correlation between performance on isolated and nonisolated pairs exceeds .25 . Since the use of four critical pairs also avoids the worry that the results might reflect peculiarities of any one pair, the inclusion of the Item variable is definitely desirable.

A somewhat different sort of simplification is based on compounding the minor variables. For instance, one Experimenter could be assigned to run the first four lists, while the second Experimenter would run the second four lists. Experimenter and Position would then form a single compound variable with two levels. This completely confounds Experimenter and Position so that significant effects of this compound variable could not be unambiguously referred to either Experimenter or Position. The effects of these variables are still controlled, however, and this confounding is often of no concern with minor variables. The technique deserves wider use, particularly when $\mathrm{N}$ is not large enough to allow a complete factorial design. It is interesting to note that Erickson did apply this technique to another minor variable not considered here.

\section{Related problems}

Partial analysis might also be applied to designs involving only major variables. However, available knowledge will not of ten clearly indicate that the interactions of major variables are negligible. In such cases, therefore, the main justification for partial analysis will be weak, and it should be used conservatively. Pooling an interaction of major variables with error is more serious than not testing it since the pooling will affect all the tests that are made. With major variables, therefore, designs with only one score per cell should generally be avoided.

There are experiments, with random factors, or with small $\mathrm{N}$, in which it is desirable or necessary to pool interactions of major variables to get adequate $\mathrm{df}$ for error. The pooling procedures that have been discussed for such cases (e.g., Bozivich, Bancroft, \& Hartley, 1956; Green \& Tukey, 1960; Paull, 1950; Winer, 1962) rely mainly on successive testing and pooling of mean squares that are not too large. These procedures have definite value in certain situations, though none of them is very satisfactory from a statistical point of view. For minor variables, pooling by prior decision is probably superior to successive testing, even in experiments with small $\mathrm{N}$ (e.g., Anderson \& Whalen, 1960).

\section{CONCLUDING COMMENT}

Statistical analysis should be viewed in its place in the complete experimental process. Partial analysis depends on personal judgment, and different investigators may arrive at somewhat different analyses of the same set of data. Although this may seem objectionable, the really critical role of personal judgment is in problems of procedure and design. The investigator who brings an additional variable under control may thereby be doing good and sufficient service even when evaluating only its main effect. 
REFERENCES

ANDERSON, N. H. Education for research in psychology. Amer. Psychol ogist, 1959, 14, 695-696.

ANDERSON, N. H. On teaching F instead of t. J. exp. Educ., 1960, 28, 261-263.

ANDERSON, N. H. Scales and statistics: parametric and nonparametric. Psychol Bull., 1961, 58, 305-316.

ANDERSON, N. H. On the quantification of Miller's conflict theory. Psychol Rev., 1962, 69, 400-414.

ANDERSON, N. H. Comparison of different populations: Resistance to extinction and transfer. Psychol. Rev., 1963, 70, 162-179.

ANDERSON, N. H., \& JACOBSON, A. F urther data on a weighted average model for judgment in a lifted weight task. Percept. \& Psychophys, 1968 , in press.

ANDERSON, N. H., \& WHALEN, R. E. Likelihood judgments and sequential effects in a two-choice probability learning situation. $J$, exp. Psychol, 1960, 60, 111-120.

BOZIVICH, H., BANCROFT, T. A., \& HARTLEY, H. O. Power of analysis of variance test procedures for certain incompletely specified models. Ann. math. Statist., 1956, 27, 1017-1043.

BROGDEN, W. J. The experimenter as a factor in animal conditioning. Psvchol. Rep., 1962, 11, 239-242.

ERICKSON, R. L. Relational isolation as a means of producing the von
Restorf effect in paired-associate learning. J. exp. PsychoL, 1963, 66, 111-119.

ERICKSON, R. L. Differential effects of stimulus and response isolation in paired-associate leaming. $J$. exp. PsychoL, 1965, 69, 317-323.

GREEN, B. F., \& TUKEY, J. Complex analysis of variance: general problems. Psychometrika, 1960, 25, 127-152.

HOEL, P. G. Introduction to mathematical statistics. (3rd ed.), New York: Wiley, 1962.

LOGAN, F. A. Incentive. New Haven: Yale Press, 1960.

PAULL, A. E. On a preliminary cest for pooting mean squares in the analysis of variance. Ann. math. Statist., 1950, 21, 539-556.

PROKASY, W. F. Inference from analysis of variance of ordinal data. Psychol Rep., 1962, 10, 35-39.

ROSENTHAL, R. Experimenter effects in behavioral research. New York: A ppleton-Century-Crofts, 1966.

SIDOWSKI, J. B., \& ANDERSON, N. H. Judgments of City-Occupation combinations. Psychon. Sci, 1967, 7, 279-280.

WINER, B. J. Statistical principles in experimental design. New York: McGraw-Hill, 1962.

\section{NOTE}

1. I wish to thank Raymond Erickson for making available the raw data reanalyzed here, as well as for valuable discussions about partial analysis. Preparation of this report was facilitated by National Science Foundation Grant GB-6666.

\section{A methodological study of auditory lateralization'}

\section{LLOYD F. ELFNER AND RICHARD T. TOMSIC, FLORIDA STATE UNIVERSITY Tallahassee, Florida 32306}

The just-noticeable-shift (JNS) threshold of a centered image was determined for four naive Ss. A two interval forced choice situation uas used under the method of constant stimuli. The three manners of presentation of the intensity imbalance were as follows: (1) an increase in intensity at one ear, (2) a decrease in intensity at one ear, (3) an increase in intensity at one ear produced concommitantly with a decrease in intensity at the opposite ear. The sign test demonstrated significant differences between method (2) and methods (1) and (3).

When two identical auditory signals with no phase difference are presented simultaneously to each ear via earphones, a single auditory image is sensed along the midline of the head. A change in intensity at one ear will cause the sound image to shift toward the ear receiving the louder or more intense signal. This phenumenon, sound lateralization, has been used to measure difference limens for intensity (Upton, 1936) and just noticeable movement of a free field sound source (Mills, 1958). The difference limen for movement in auditory lateralization has been termed just-noticeable-shift or JNS.

The procedure used in the determination of JNS is to have the listener judge the instances when the centered sound inage moves to one side as a result of a change in intensity at one ear. The image can be shifted from the center toward a specific side by either increasing the intensity at one ear or by decreasing the intensity at the other ear. A third way of inducing such a shift is by a combination of the above. That is, the intensity at one ear is increased at the same time that the intensity at the other ear is decreased. This method of presentation differs from the others in two ways. First, the overall intensity level of the signal presented to the listener remains relatively unchanged, hence, not confounded with the shift in lateralization. Second, a varying signal is presented simultaneously to the two ears rather than to a single ear.
The present study is an attempt to determine whether the three methods of presentation cited above produce similar difference limens for JNS from a center position.

\section{METHOD}

Subjects

Four, paid, undergraduate students enrolled in Introductory Psychology courses at Florida State University who could readily lateralize a sound image were employed in the study. All Ss exhibited clinically normal hearing (ISO standards). The Ss were given extended practice in detection of shift from the center of a binaurally produced image.

\section{Procedure}

A block diagram of the apparatus appears in Fig. 1. The tone, produced by an audio-oscillator (Hewlett-Packard, model 204B), was passed through electronic switch No. 1 (Grason-Stadler, model $829 \mathrm{C}$ ) which controlled the onset and offset of the overall signal. A 10-msec rise-decay time was programmed for all signal presentations. The signal was then led through a phase shifter (Grason-Stadler, model E3520B) which served both as a signal splitter and as a compensator for phase differences in the signals led to the earphones. The signals were further split by transformers with one signal from each transformer being passed through precision attenuators (Altec-Lansing, model DA8736, 38 40) which were adjustable to $0.1 \mathrm{~dB}$. These signals were led to the "A" input on both channels of electronic switch No. 2 (Grason-Stadler, model $829 \mathrm{C}$ ). These were the test signals that were increased or decreased as the procedure required. The remaining signals from the splitting transformers were led directly to the "B" inputs of electronic switch No. 2 and were employed as the "center" or standard signal.

When switch No. 1 was turned on, switch No. 2 had the " $B$ " (standard) signal on. This was followed by a change to the " $A$ " (test) signal. After switch No. 2 was in the "A" position, switch No. I turned the signal off. Two electronic timers (GrasonStadler, model 471) were used to program the signal durations. A 